\title{
Uber neue Artefaktfunde aus der Heidelberger Stufe
}

\author{
Von Alfred Rust, Ahrensburg (Holstein)
}

Mit 5 Abbildungen im Text

$\mathrm{Z}$ u s a m menf as u ng. Nach der Entdeckung altpleistozäner Artefakte der Heidelberger Stufe in Norddeutschland und bei Mauer ließ sich der erkennbare Lebensraum des Homo heidelbergensis jetzt wesentlich ausweiten. Neue Funde aus den präglazialen Terrassen von Süßenborn bei Weimar erwiesen eine Besiedlung auch Mitteldeutschlands, und gleichartige Funde aus den ältesten Donauterrassen bei Wien werden als Hinweis auf eine weitgehend nach Osten in den Kontinent hineinreichende Besiedlung durch den Heidelberger Menschen aufgefaßt.

Artefaktfunde aus den Rheinschottern bei Brüggen führen an die plio-pleistozäne Grenze. Im Nachtrag werden Heidelberger Artefakte aus den oberpliozänen Schottern von Sülzfeld in Thüringen angeführt, die den Artefakten von Mauer typologisch sehr nahestehen.

$\mathrm{Su} \mathrm{m}$ ma ry. After Lower Pleistocene artifacts of the Heidelbergian had been discovered in North Germany and near Mauer, the traceable living space of Homo beidelbergensis could now be enlarged essentially. Recent finds unearthed from the pre-glacial terraces of Süssenborn near Weimar furnished evidence of his settling in Central Germany, too, and finds of the same kind discovered within the oldest terraces of the Danube River near Vienna are interpreted to be a hint of the Heidelberg man having settled far eastwards on this continent

Artifacts found within the Rhine gravels near Brüggen lead to the Plio-Pleistocene boundary. The addenda enumerate Heidelbergian artifacts traced in the Upper Pliocene gravels of Sülzfeld in Thuringia, which closely approach the artifacts of Mauer typologically.

$\mathrm{R}$ é $s$ u m é. Après la découverte d'ustensiles du Heidelbergien dans des gisements du Pléistocène inférieur en Allemagne du Nord et dans le voisinage de Mauer, il fut maintenant possible d'élargir essentiellement le domaine retraçable du Homo heidelbergensis. De nouvelles trouvailles dans les terrasses préglaciales de Süssenborn près de Weimar ont prouvé qu'aussi l'Allemagne centrale avait été habitée par lui, et des trouvailles semblables dans les terrasses les plus anciennes du Danube près de Vienne peuvent indiquer que l'homme heidelbergien ait poussé sa colonisation assez loin dans l'est du continent.

La trouvaille d'ustensiles dans les graviers du Rhin, non loin de Brüggen, conduit à la limite plio-pléistocène. Le supplément énumère des ustensiles heidelbergiens trouvés dans les graviers du Pliocène supérieur à Sülzfeld en Thuringe, qui, typologiquement, sont très proches des ustensiles de Mauer.

\section{E in le it ung}

In einer kürzlich vorgebrachten informativen Beschreibung altpleistozäner Artefakte (Kust 1956) wurde u. a. auch auf die bisher erkennbare Begrenzung des Lebensraumes des Homo heidelbergensis als Träger solcher altpleistozänen Werkzeuge hingewiesen. Die Abgrenzung war durch die Fundvorkommen in der westlichen Hälfte Deutschlands gegeben. Dieses Gebiet zwischen der Ostsee und dem Fuße der Alpen liegt östlich des südwesteuropäischen Brückenkopfes der Faustkeilkulturträger afrikanischen Ursprungs. Die kontinentwärts zu Frankreich und Südengland gerichtete Lage ließ vermuten, daß der Heidelberger als 'Träger einer selbständigen, mit den Faustkeilindustrien nicht verwandten Kultur der Bewohner auch der innerkontinentalen Gebiete während des Frühpleistozäns war. Wir schlugen eine intensivere artefaktbezügliche Beobachtung von altpleistozänen Ablagerungen in den östlich des Rheines gelegenen Gebieten vor. Die Sicherung dieser Auffassung erhielt kürzlich eine wesentliche Festigung.

1. Artefakte aus den altpleistozänen Donauterrassen bei Wien

Im Anschluß an die Tagung der Deutschen Quartärvereinigung in Laufen am 4. Sept. 1955 besuchten die Exkursionsteilnehmer auch Wien. Dort bat mich Herr Dr. Freising um die Begutachtung von vermutlichen Artefakten aus altpleistozänen Donauschottern, die Herr Professor H. MoHr seit Jahren gesammelt hatte. Unter den 
yon Prof. MoHr vorgelegten Objekten befanden sich zweifelsfreie Artefakte der Heidelberger Stufe. Eine zeitliche Beschränkung erlaubte die spezifizierte Durchsicht der Sammlung nicht, aber ich glaube mich in der Erinnerung nicht zu irren, dort mindestens zwei Dutzend typische Artefakte gesehen zu haben.

Am 10. Sept. führte eine Exkursion u. a. in den Aufschluß Rudolfsziegelöfen der frühpleistozänen Donauschotter am Laaer Berg bei Wien. Im Anschluß an die einleitenden Worte der Wiener Kollegen dortselbst glaubte ich zur „Rehabilitierung“ der MoнRschen Funde auf die Tatsache des Vorhandenseins von Artefakten in den ältesten Wiener Schottern hinweisen zu müssen. Herr Kollege H. Schwabedissen hatte das Glück, aus den Schottern ein vorzügliches Gerät auflesen zu können, das wir mit freundlicher Erlaubnis der Wiener Kollegen und Herrn Schwabedissen's in Abb. 1 wiedergeben.

Frau Dr. Maria Motru, die sich seit Jahren ebenfalls für die Artefaktnatur eines Teils des Fundgutes aussprach, war so freundlich, mir in Graz einige weitere Artefakte dieser Art aus der Mohr'schen Sammlung vorzulegen, und Herr Direktor Dr. KüPPER machte mir einige von ihm selbst gefundene vorzügliche Werkzeuge zugänglich, u. a. das Stück Abb. 2 und 3.

Die hervorragende Bedeutung der Wiener Artefakte scheint uns in der Tatsache zu liegen, daß mit deren Auffinden eine wesentliche Erweiterung des Lebensraumes des Heidelbergers in den Kontinent hinein gesichert ist. Wien liegt östlich des Mittelpunktes der Luftlinie Paris-Schwarzes Meer! Wir sind auf Grund verschiedener hinsichtlich der Ausbreitung pleistozäner Kulturgruppen gewonnener Erkenntnisse der Auffassung, daß es gelingen wird, in den Schottern der Donau bis zur Mündung und über diesen Raum auch ostwärts hinaus fündig zu werden.

Eine Beschreibung der Wiener Fundstücke mit allen weiteren Gegebenheiten wird durch Prof. Mohr und Frau Dr. Motrl erfolgen.

\section{Artefakte aus den altpleistozänen Ilm-Terrassenschot tern} von $S$ ü $B$ en born

Im November 1954 konnte ich eine erstmalige Einsicht in die Schottergruben von Süßenborn bei Weimar nehmen, wobei außer einem nicht völlig klar ausdeutbaren Hobel keine Artefakte nachgewiesen werden konnten. Von besserem Erfolg begleitet war ein zweiter längerer Besuch am 15. 11. 1955 in Begleitung von Herrn Dr. H. D. KaHLKE. Ich konnte 3 Artefakte auffinden. Für die Möglichkeit, dic Stücke hier abbilden zu können, sind wir Herrn Prof. G. Венм-Blancke, Weimar, sehr dankbar.

Bei diesen Artefakten handelt es sich erstens um einen Nasenschaber mit gegenständiger Retusche. Das Stück besteht aus Porphyrit, es ist mäßig abgerollt. Die Unterseite des Gerätes läßt in Abb. 4 deutlich werden, daß die beiden Hohlkerben übersteilt geschlagen sind. Diese Buchten sind also nicht so zugeschlagen, wie sie der Vorzeitmensch herrichtete, wenn er damit arbeiten wollte. Sie sind, wie für die Heidelberger Stufe typisch, verstumpft und dienten lediglich zur Herausarbeitung des nasenartigen Vorsprunges, mit dem dann gearbeitet wurde. Die Nase ist oben in typischer Art gegenständig zu den Buchten retuschiert. Die Oberseite des Gerätes gibt Abb. 5 wieder, gleichfalls die Seitenansicht, aus der die Höhe der sehr steilen Retusche und der für die Heidelberger Industrie typische trapezoide Querschnitt des Gerätes deutlich wird.

Das zweite Gerät ist ein stark gerollter kurioser doppelter Nasenschaber. Das plattige Artefakt besteht aus Porphyr und ist an den Spitzenenden nicht gegenständig retuschiert.

Auch das dritte Gerät ist wie die eben genannten aus einem Geröllstück angefertigt. Es ist als Hohlschaber mit kleinem nasenartigem Vorsprung zugerichtet. Die 

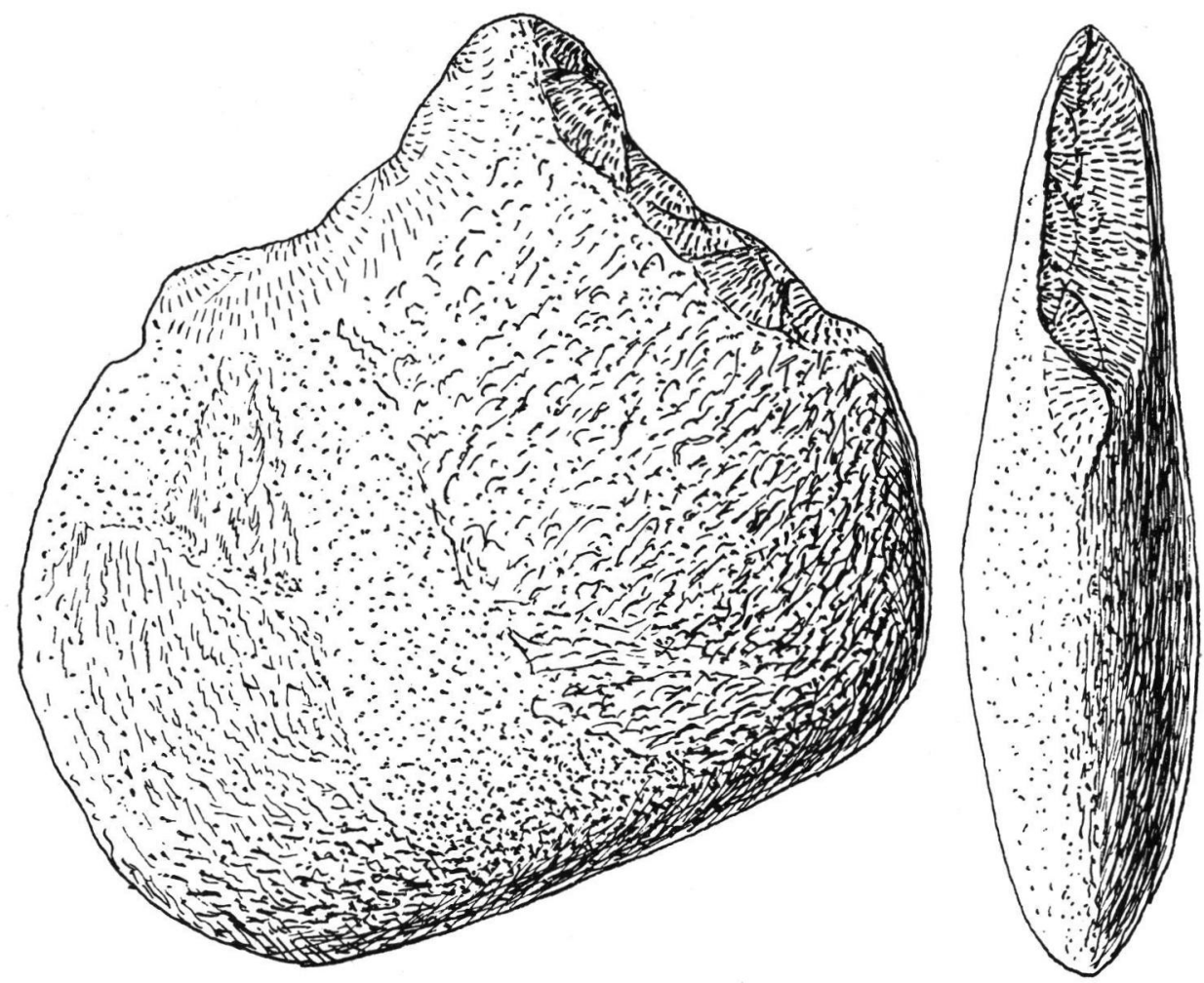

Abb. 2. Artefakt vom Heidelberger Typ (Ober- und Seitenansicht) aus den Schottern der Donauterrassen bei Wien. Nat. Gr.

große Kerbe ist übersteilt geschlagen, so daß es sich möglicherweise auch um einen kurzen Nasenkratzer handelt.

Die genannten 3 den ausgesiebten Schottern entnommenen Artefakte von Süßenborn lassen sich der Heidelberger Kultur angliedern. Sie verdeutlichen wahrscheinlich eine jüngere Phase dieser Industrie.

Úber'das tatsächliche Alter der Süßenborner Schotter konnte von der geologischpaläontologischen Seite bisher noch keine einheitliche Auffassung erzielt werden. Die altpleistozäne Zeitstellung ist aber nie angezweifelt worden. Diese Datierung basiert in erster Linie auf der Tatsache, daß die "präglazialen Schotter" (SoERgel 1924) noch nicht, wie die jüngeren Ablagerungen der Ilm, Geschiebe nordischen Ursprungs führen. Während der Ablagerung hatte somit noch kein Gletschervorstoß das Zuzugsgebiet der Ilm erreicht.

Diese Süßenborner Ablagerungen aus dem Grenzgebiet des fennoskandinavischen Vergletscherungsbereiches sind für das Verständnis einer erfolgreichen Forschung nach Artefakten des Heidelbergers inmitten des Moränenaufschüttungsgebietes recht lehrreich.

Die Ur-Ilm floß nach Norden ab und mündete in einen größeren Strom, der, zum Atlantik abfließend, das spätere norddeutsche Moränengebiet durchzog. Die Ablagerungen dieses „Ur-Stromes“, der vor dem Anlaufen der Mindel-Vereisung noch kein nordisches Material führte, wurden später vom Eise überfahren. Dabei wurden die Schotter mit den aus kristallinen Gesteinen Mitteldeutschlands hergestellten Artefakten aufge- 


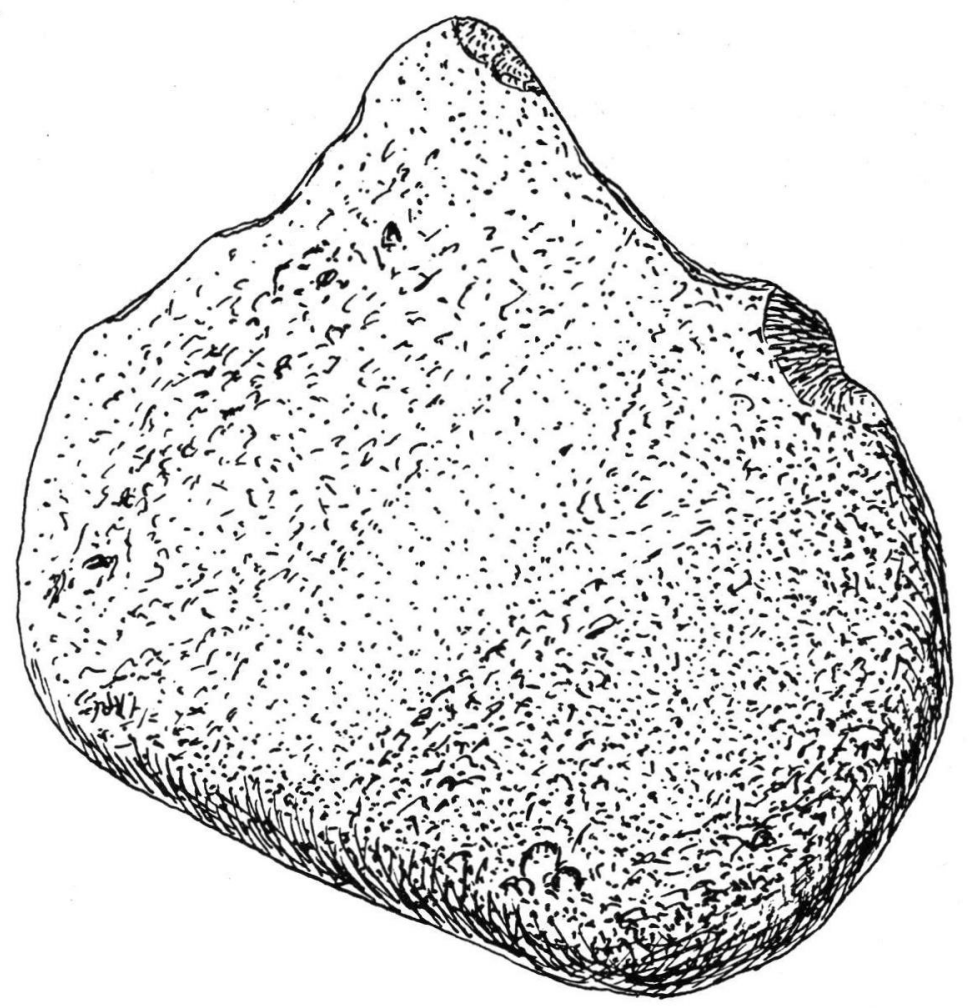

Abb. 3. Ansicht der Unterseite des Artefaktes von Abb. 2. Nat. Gr.

nommen und verfrachtet. Nach dem Niederschlagen lagen dann also diese Artefakte inmitten einer Ablagerung, die u. a. vorzüglichen nordischen Flint führt (vgl. Rust 1956).

Diesen „Ur-Strom“, der einen Teil der heutigen Ostseesenke durchzogen haben mag, lassen wir hier rein theoretisch der Elbe gleichlaufen. Es ist zu erwarten, daß wir nördlich dieser Elbe nur solche Heidelberger Artefakte auffinden werden, die aus skandinavischem Material angefertigt worden sind, während in den Moränengebieten südlich der Elbe bis nach England hinein zusätzlich Artefakte erwartet werden können, die aus in Mitteldeutschland anstehendem Material gefertigt wurden.

\section{A rtefakte aus den "ältestdiluvialen Schottern “ des $\mathrm{Nieder} r$ heines im Ra me Brüggen}

Am 3. 5.56 besuchte ich erstmalig zwei der u. a. von R. Woltens (1954) beschriebenen Aufschlüsse im Raume Brüggen im deutsch-holländischen Grenzgebiet. Es wird dort tertiären Sanden aufliegender "Reuverton" abgebaut, der von mehrere Meter mächtigen „ältestdiluvialen Schottern“ (zumeist Feinsanden) bedeckt ist. Diesen liegt „Tegelenton“ (oberes Villafranchiano) auf, während das Hangende aus Sanden der „Hauptterrasse“ des Rheines besteht. U. REIN (1955) konnte durch Pollenanalysen den Reuverton dem oberen Pliozän und den Tegelenton einem frühen Wärmeabschnitt des Frühpleistozäns zuweisen.

In der erstbesuchten Grube (Grube Brüggener A.-G.) wird nur Reuverton abgebaut. Am Fuße der etwa $5 \mathrm{~m}$ hoch über dem Ton anstehenden Sande fand ich 2 vorzügliche 


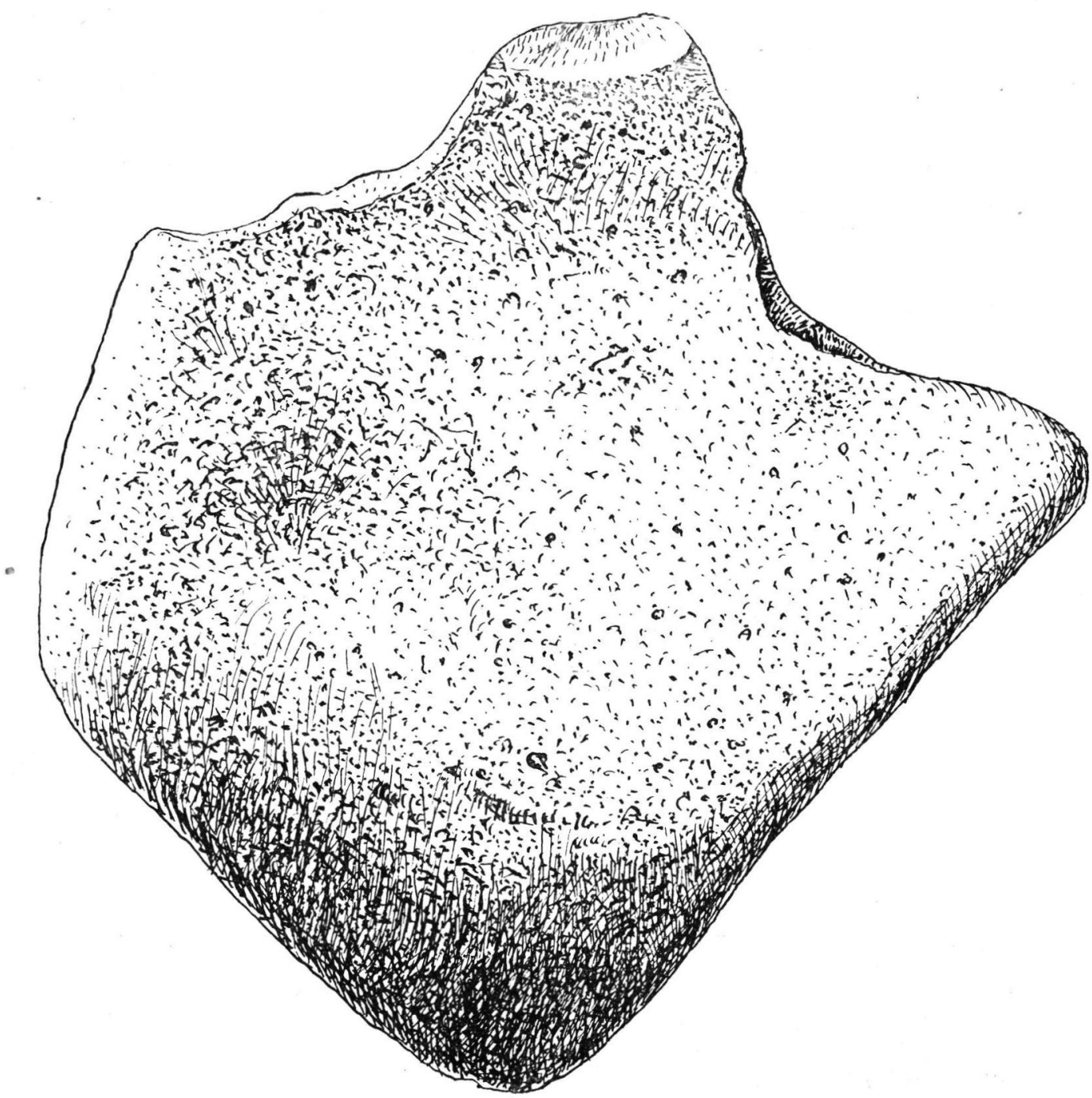

Abb. 4. Großer Nasenschaber aus den „präglazıalen Schottern“ von Süßenborn bei Weimar.

(Unterseite). Nat. Gr.

Heidelberger Artefakte, die sekundär auf dem Ton lagen. Es handelt sich um einen Nasen- und um einen Seitenschaber. Die Sande wurden direkt an der Fundstelle durch einen Bagger abgebaut, und die Fundumstände lassen vermuten, daß die Artefakte aus den Sanden stammen.

In der anschließend besuchten Grube (Gebr. Laumanns) wird Reuver- und Tegelenton abgebaut. An der Oberfläche des getrenntliegenden Abraumes der ältestdiluvialen Schotter fanden sich 6 Heidelberger Artefakte, auf dem Abraum der Hauptterrasse ein gleichartiges Artefakt. Herr Dr. Woltens wies mich später freundlichst darauf hin, daß trotz des voneinander abgetrennten Abbaues der Abraum der ältestdiluvialen Schotter leichte Verunreinigungen durch den Abraum der Hauptterrassenschotter aufweisen 


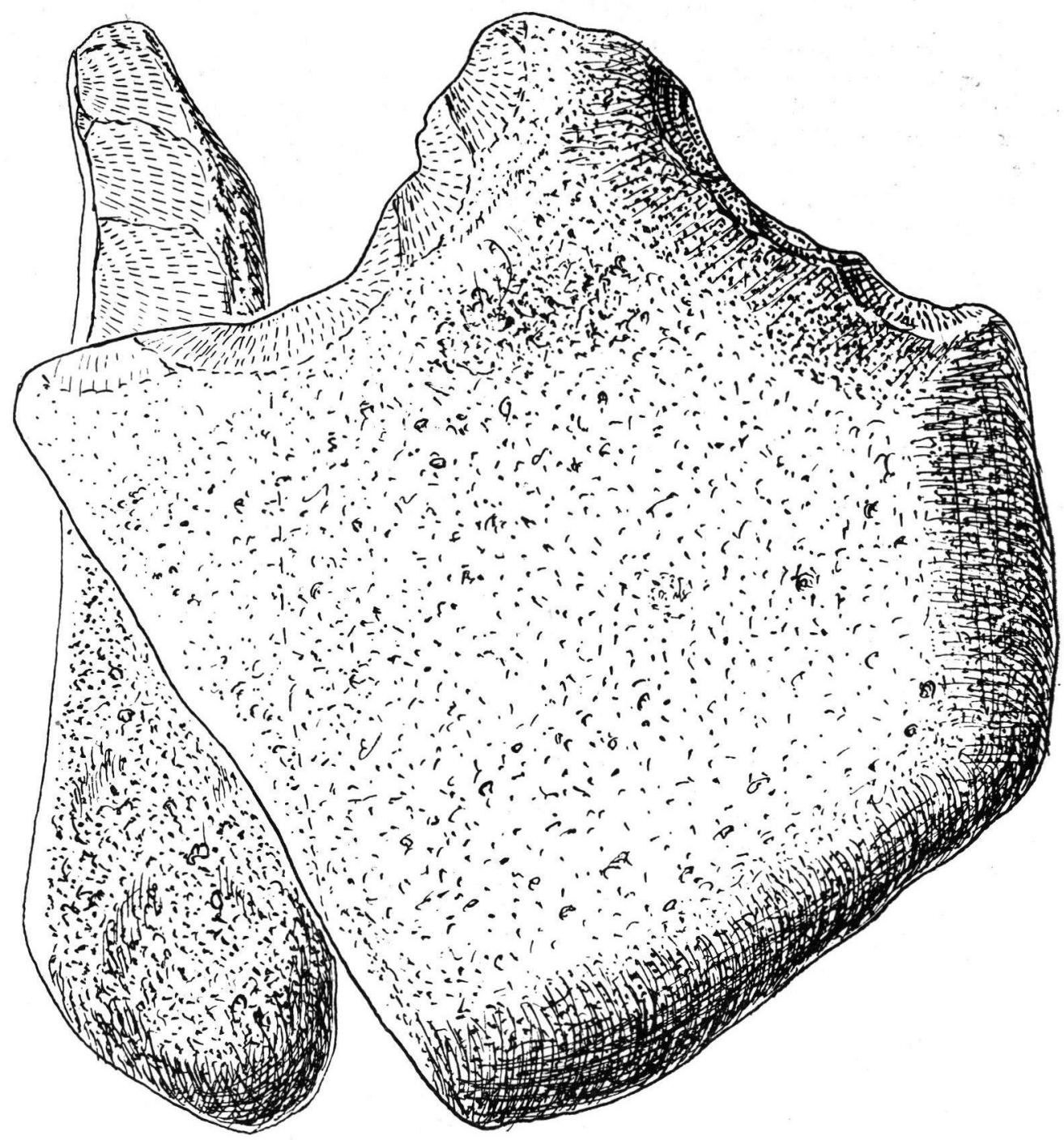

Abb. 5. Ober- und Seitenansicht des Artefaktes von Abb. 4 aus Süßenborn. Nat. Gr.

könne. Ob die ältesten Schotter (wie wir als sicher ansehen) tatsächlich Artefakte führen, wird sich durch die Untersuchung des Inhaltes der von R. Wolters beschriebenen, in den tertiären Ton eingeschnittenen Bachbetten erweisen lassen.

Einige weitere Artefakte entstammen einer Sandgrube bei Brüggen, die in den Ablagerungen der Hauptterrasse angelegt ist. Fast alle eben genannten Artefakte sind wie diejenigen aus dem nordischen Moränengebiet leicht windgeschliffen oder als Windkanter facettiert!

Soweit ersichtlich, sind wir mit den Brüggener Artefakten bis zur plio-pleistozänen Grenze hinuntergelangt, denn die ältestdiluvialen Schotter werden als vorgünzzeitlich angesprochen. 
IV. Über die Alterstellung der Heidelberger Kultur

Zur Frage der Datierung der Heidelberger Stufe ist es eine Voraussetzung, daß wir für den Träger dieser Industrie, den Homo beidelbergensis, eine, ,biologische Lebensdauer" in einer Größenordnung von 100000 Jahren oder ein Mehrfaches dieser Zeit annehmen müssen. Diese Zahlenangaben sind auf das Altpleistozän und weiter hinab auf die jungpliozänen Zeitabschnitte zu beziehen. Wir möchten also zum Ausdruck bringen, daß die Lebensdauer der Heidelberger Industrie nicht auf einen begrenzten Zeitabschnitt, sagen wir auf die Dauer des Günz-Mindel-Interglazials, begrenzt werden darf.

Um eine werkzeugtechnisch so klar und fest umrissene Industrie erwachsen zu lassen, wie sie z. B. aus Mauer vorliegt, bedurfte es in der Frühzeit der Menschheitsentwicklung einer Anlaufzeit von einigen Hunderttausend (oder gar von Millionen) Jahren. Als Gegenstück zu dieser Auffassung möchten wir in Erinnerung rufen, daß sich z. B. der Faustkeil vom Günz-Mindel-Interglazial ab bei leichter Modifizierung über fast eine halbe Million Jahre bis ins Mittel-Würm hinein in den Grundformen gehalten hat.

Wir bringen diese kurzen Ausführungen hier auch in der Hoffnung vor, dadurch eine größere Aufgeschlossenheit bei den Kollegen von der Geologie zu erreichen, verbunden mit der Bitte, nebenher vor allem auch in pliozänen Aufschlüssen ein Augenmerk auf Artefakte vom Heidelberger Habitus zu richten. Alle altpleistozänen Werkzeuge von Wien z. B. sind von Geologen entdeckt worden!

Nach den bisher gemachten Erfahrungen sind es in den eisfrei gebliebenen Gebieten vor allem die Flußterrassen, die eine Möglichkeit für die Datierung der Heidelberger Kultur bieten. Allem Anschein nach werden für den Donau-Raum die Terrassen bei Wien in Anbetracht ihrer Vielzahl einmal eine erstrangige Bedeutung in dieser Beziehung gewinnen. Desgleichen diejenigen im Raume um Budapest.

In Mauer konnten nach der ersten Beschreibung der Fundvorkommen (Rust 1956) weitere vorzügliche Werkzeuge geborgen werden, die sich alle dem Rahmen unserer Industrie, die keine Bifacegeräte kennt, einpassen. Als Neuerscheinung konnten wir den Typ der geschränkten Säge feststellen. Das Alter der Ablagerungen in der Grube Grafenrain bei Mauer ist umstritten, und die Datierungen schwanken zwischen dem Günz-Mindel-Interglazial und einem Mindel-Interstadial.

Im Bereiche des nordischen Vereisungsgebietes konnten wir zahlreiche neue Artefakte auffinden, so daß uns heute ein Studienmaterial von mehreren tausend Geräten der Heidelberger Stufe zur Verfügung steht.

Erstmalig gelang es uns nun auch, Heidelberger Artefakte an der Oberfläche des außersten Bereiches der Würmmoränen und in diesen im Raume Hamburg-Ahrensburg autzufinden. Es sind das bisher über 2000 vorzügliche Typen, die fast alle windgeschliffen sind. Es wurden darüber hinaus aber auch Fundstücke dieser Art sowohl an der dänischen Grenze als auch im Grenzbereich Schleswig-Holstein-Mecklenburg festgestellt. G. H. BrüCKner fand Heidelberger Artefakte in Würm-Grundmoränen an der Ostseeküste.

Diese Befunde sind nicht verwunderlich und bestätigen nur fester die Erkenntnis, daß viele Moränen u. a aufgenommene Bestandteile der vorangegangenen Vereisung oder solche von noch älteren Ablagerungen enthalten.

Damit werden aber für die Geologie denkbare Schwierigkeiten für exakte Auswertungen von Ergebnissen der Geschiebezählung angedeutet. Die Heidelberger Artefakte finden sich (als Bestandteile altpleistozäner oder oberpliozäner Ablagerungen) im Bereich der Würmmoränen z. T. in starker Konzentration auf engstem Raum. Wir führen als Beispiel vorerst nur zwei kleine getrenntliegende Ackerstücke aus unserem begrenzten Arbeitsgebiet an, von deren Oberflächen jeweils rund 1000 Heidelberger Artefakte abgesammelt werden konnten. Welche Mächtigkeit diese „Fundschichten“ aufweisen, 
konnte noch nicht ermittelt werden. Im Gegensatz dazu wurde z. B. in einer nahegelegenen Kiesgrube unter den zu Bergen angehäuften unzählbaren Geschieben nicht ein einziges Artefakt aufgefunden. Diese Ablagerung könnte somit vielleicht einen "reinen“ Geschiebebestand aus dem Zuzugsgebiet dieser Würmmoräne aufweisen.

Eine solche Darstellung der Verhältnisse muß vorerst befremdlich wirken, und wir stehen auch erst am Anfang der Erkenntnisse auf diesem Gebiet. Aber es hat den Anschein, daß wir auch auf diesem Sektor noch zu genaueren Einblicken in den tatsächlichen Ablauf der diesbezüglichen glazialen Geschehnisse gelangen werden. Es glückte uns nämlich z. B. kürzlich, im Bereich der Würmmoränen (in der Würmrandlage A 4 nach GRIPP, 1924) eine „Mousterien-Kulturschicht" aufzufinden, die ein Mittelpaläolithikum von levalloisartigem Charakter mit u. a. vorzüglichen La Quina-Typen enthält. Das Vorkommen dieser „Hagener Gruppe“ kann auf einige tausend Artefakte geschätzt werden. Die Geräte liegen $5 \mathrm{~m}$ unter der heutigen Oberfläche, sie sind konzentriert angereichert und gebrauchsfertig scharfkantig erhalten geblieben.

Die Tatsache, daß diese nachweisbar vom Eise transportierten Artefakte scharfkantig sind und konzentriert liegen, möchten wir als einen tatsächlichen Hinweis für unsere Auffassung herausstellen, daß auch altpleistozäne Kulturreste vom Eise aufgenommen, geschlossen in Schüben transportiert und bei relativ guter Erhaltung in konzentrierter Anreicherung abgelagert sein können. Wir erinnern in diesem Zusammenhang an die in einer rißzeitlichen Grundmoräne gelegenen Artefakte der Altonaer Stufe, die z. T. in Originalschärfe auf uns gekommen sind (RUst 1956).

Die Artefakte der wohl frühwürmzeitlichen "Hagener Gruppe“ von Ahrensburg sind zu mehr als 95\% aus Quarzit, Granit und ähnlichem kristallinen Gesteinen hergestellt. Diese Tatsache wirkt im Bereiche des nordischen Moränengebietes im „Feuersteinland" befremdlich. Wenn wir aber auf Grund der Geschlossenheit der Artefaktvorkommen annehmen, daß das blockpackungsartige Material der Schicht, in der die Werkzeuge heute liegen, jenes Material ist, das am ehemaligen Wohnplatz vorhanden war, so hatte der eiszeitliche Jäger am Orte fast keine Möglichkeit, Flintwerkzeuge herzustellen. Der Anteil verwendbaren Flintes von vielleicht Faustgröße ab liegt beim laufend abgebauten Material höchstens um 1\%. Ob dazu dieses geringe Flintvorkommen, als an der ehemaligen Oberfläche gelegen, dem Werkzeugfertiger auch tatsächlich zugänglich war, ist zweifelhaft. Bei anderen nahegelegenen Kulturvorkommen dieser Art liegt der Anteil der Flintgeräte entsprechend den höheren Anteilen von Flintknollen im Geschiebebestand höher.

Das Vorkommen der Hagener Gruppe gibt uns wiederum einen Hinweis, daß in Nordeuropa Artefakte durch Eistransport (das gilt auch für die Mindel- und Rißglaziale) nicht immer zerstört und weitläufig verstreut wurden, und es gibt uns ferner ein Beispiel dafür, daß wir vielleicht durch gar zu starre Blickrichtung auf den Feuerstein Kulturreste übersehen haben, die bezeugen könnten, daß Nordeuropa z. B. auch im Mittelpleistozän weitaus lebhafter besiedelt war, als wir bisher glaubten annehmen zu können.

Unser Befund ist jedoch für allgemeine siedlungsgeschichtliche Probleme von größerer Bedeutung. Wir sind nach mancherlei Erfahrungen der Ansicht, daß im Bereiche der skandinavischen Vereisung an der Oberfläche fast aller Moränen Artefakte des Heidelbergers aufgefunden werden können und, jeweils gestuft, auch solche aus jüngeren Industrien. Auf Grund der genannten Befunde müßten uns somit z. B. Faustkeile, sofern solche im norddeutschen Flachland hergestellt worden wären, zweifelsohne längst bekanntgeworden sein. Wir können unter diesen Umständen somit als indirekt erwiesen ansehen, daß Nordeuropa, sagen wir nördlich der Elbe, zu keiner Zeit vor dem RißWürm-Interglazial festes Jagd- und Wohngebiet der Träger von Faustkeilkulturen 
afrikanischen Ursprungs gewesen ist. Diese Auffassung schließt gelegentliche kurzfristige Jagdzüge nicht aus.

Für eine einengende Datierung der Heidelberger Funde oder einen Teil derselben ließen sich im schleswig-holsteinischen Raum über die in der zitierten Arbeit stratigraphisch und materialmäßig erschlossenen Erwägungen hinaus bisher keine weiteren Festpunkte gewinnen. Möglicherweise gelingt es aber im Zuge dieser ersten Erkundungen weiter im Süden und zwar im Grenzbereich des nordischen Vercisungsgebietes voranzukommen, denn dort finden sich im Gegensatz zu Schleswig-Holstein „reine“ Moränenrückstände ältester Vereisungen.

Für Erwägungen dieser Art können wir als praktisches Beispiel die Verhältnisse von Süßenborn heranziehen. Diese Schotter aus den nach SoErgel "präglazialen“ Terrassen sind, wie gesagt, nicht vom Eise überfahren und aufgenommen worden. Der Vorgang einer Aufnahme und eines Transportes kann aber für den unteren Lauf der nach Norden abfließenden "Ur-Ilm" und eines „Urstromes" als fraglos geschehen erachtet werden. Es besteht somit theoretisch und praktisch die Möglichkeit, daß die in Süßenborn gelegenen Schotter und die in diesen vorhandenen "primär gelegenen“ Artefakte einige Kilometer nördlich von Süßenborn „sekundär" gelagert, verstreut im Moränenschutt liegen.

$\mathrm{Da}$ diese in den Moränen gelegenen Artefakte älter als die Ablagerung sein müssen, ergäbe sich ein höheres Alter, als es die vielleicht datierbare Moräne selbst besitzt.

Die im weiteren Verlauf östlich des Harzes am weitesten nach Süden vorgeschobe nen Moränen sind z. T. mindelzeitlich (Woldstedt 1950), und wenn es gelingt, in diesen Artefakte aufzufinden, so sind sie zumindest älter als ein jüngster Mindelvorstoß.

Für den Schleswig $\vdash$ Holstein am nächsten gelegenen Raum erhoffen wir uns Resultate für die Datierung im engbegrenzten Mindel-Moränengebiet westlich des Harzes, das durch G. Lürtig (1954) kürzlich eingehend bearbeitet worden ist. Auch in diesem Grenzgebiet gelingt es vielleicht, vor dem Eisrand Heidelberger Artefakte in "primärer“ Lage in Flußschottern und, "sekundär“ gelegen, die gleichen Typen in den Endlagen der Moränen aufzufinden.

Auf diesem Wege mag es in der Zukunft auch einmal gelingen, einen gewissen Aufschluß über den Streubereich des Ausgangsmaterials für die Herstellung von Werkzeugen des Urmenschen, d. h. also für das im Harz, im thüringischen Raum usw. anstehende Material, zu gewinnen. Denn die Verwendung von Flint im Gesamtbereich der nordischen Vereisung setzte erst nach der Mindel-Vereisung ein. Die schleswig-holsteinischen Artefakte im Umfange von mehreren Tausend Stücken bestehen annähernd zu 100\% aus ostschwedischen Gesteinen oder z. B. aus Porphyr vom Grunde der östlichen Ostsee.

\section{Abschließende Betrachtungen}

Durch neue Artefaktfunde konnte der bisher durch die Vorkommen von Mauer und Schleswig-Holstein erkennbare schmale Lebensraum des Homo heidelbergensis als wesentlich ausgedehnter nachgewiesen werden. Als den von diesen Urmenschen besiedelten Bezirk können wir heute ansehen: den Strombereich großer frühpleistozäner Flüsse Nordund Mitteldeutschlands einschl. des Ostseegebietes, des Rheins und der Donau bis hinab nach Wien.

Damit zeichnet sich jetzt ein wesentlich erweiterter Lebensraum des Heidelbergers ab Dieses Gebiet liegt kontinentwärts vom atlantischen Siedlungsbereich der Faustkeilkultur. Der Ausbreitung des Heidelbergers waren nach Osten zu keinerlei klimatisch bedingte Grenzen gesetzt oder Hindernisse in Form von Meeresarmen oder hohen Gebirgszügen in 
den Weg gestellt. In östlicher Richtung wohnten im Kontinent, soweit wir orientiert sind, auch keine „fremden Völker“, etwa Faustkeilträger. Es gibt wohl kein Argument gegen die Annahme, daß z. B. die Donau im ganzen Strombereich von Heidelbergern besiedelt gewesen sein sollte. Zusammenfassend möchten wir also nochmals unsere Auffassung unterstreichen, daß neben Westeuropa auch das ganze kontinentale Europa, den Balkan eingeschlossen, der Pontische Raum und wahrscheinlich auch große Gebiete Nordund Westasiens, sofern sie nicht wildarme Wüstengebiete waren, vom Homo beidelbergensis bewohnt wurden.

Auf Grund der Tatsache, daß uns jetzt Tausende dieser altpleistozänen Artefakte des Heidelbergers vorliegen, ist es möglich, ein festgefügtes Typenbild von dieser Kultur aufzustellen. Die Artefakte von Sylt, Hamburg, Brüggen, Süßenborn, Mauer und Wien bilden typenformlich jene Einheit, wie wir sie kürzlich beschrieben haben. Die formliche Geschlossenheit der Industrie spricht für einen einheitlichen Träger dieser Kultur, den wir im Homo beidelbergensis sehen.

Die Steinindustrie des Heidelbergers ist von der des Faustkeilträgers weitgehend (oder gar grundlegend) verschieden. Blickfangend ist bei den Faustkeilträgern die Anwendung der Bifacetechnik, die der Heidelberger Kultur völlig fremd ist. Ob aus diesen werkzeugtypologischen Unterschieden auch auf eine anthropologische $Z_{\text {weigliederung }}$ der jeweiligen Träger der Industrien geschlossen werden darf, ist noch ungewiß. Soweit der Literatur zu entnehmen ist, werden die ältesten Faustkeilkulturen ins GünzMindel-Interglazial gestellt. Sie sind in Europa also jünger als die Geröllkultur des Heidel̆bergers, z. B. aus den ältestdiluvialen Schottern von Brüggen. Die frühpleistozäne Industrie des Heidelbergers ist als in Europa bodenständig aus Vorformen erwachsen anzusehen, während die Faustkeilkulturen als aus Afrika nach Europa eingewandert zu erachten sind.

Ahnliche Verhältnisse liegen in Afrika vor. Auch dort sind die Geröllkulturen (pebble tool-Kulturen), so das Kufuan, älter als die Bifaceindustrien. Ob letztere in Afrika aus den Geröllkulturen erwachsen sind, konnte aus den bisherigen Materialfunden anscheinend noch nicht mit Sicherheit erwiesen werden. Fand ein solcher Entwicklungsvorgang nicht in Afrika statt, so könnte man, da Europa für solche Wandlungen ebenfalls ausfällt, vielleicht an eine Invasion „prä-chopping tool-artiger Kulturträger" denken, die, von frühpleistozänen Vereisungserscheinungen bedrängt, aus Asien nach Afrika eingewandert sein könnten. Haben sich solche Vorgänge tatsächlich abgespielt, so wurde Europa von dieser frühesten Invasion im Sinne einer Ostwest-Stoßrichtung ebensowenig betroffen wie von einer späteren Invasion der Faustkeilträger aus dieser Richtung.

Herr Dr. G. Smolla machte mir kürzlich eine Anzahl pebble tools zugänglich, die er als Teilnehmer des Afrika-Kongresses 1955 in Ostafrika aufgesammelt hatte. Diese ersten mir vorgelegten Originale erwiesen sich als den Heidelberger Artefakten außerordentlich ähnlich. Wir konnten feststellen, daß die stratigraphisch ältesten pebble tools sich mit den Heidelberger Artefakten typologisch fast völlig decken. Inwieweit das auch für die Gesamtindustrie der ältesten pebble tools zutrifft, wäre bei der Untersuchung einer größeren Anzahl afrikanischer Funde festzustellen.

$\mathrm{Ob}$ auf Grund einer sehr nahen artefakttypologischen Übereinstimmung auch auf einen einheitlichen Träger der frühpleistozänen Geröllkulturen Europas und Afrikas geschlossen werden kann, entzieht sich noch unserer Kenntnis. An eine solche auch anthropologische Bindung kann aber, zumindest für einen Teil der afrikanischen Urbevölkerung, gedacht werden.

Wenn sich die Fundidichte von altpleistozänen oder oberpliczänen Artefakten, wie wir sie auf engstem Raum bei Hamburg erarbeiteten, als für das ganze nordische Moränengebiet gültig erweisen sollte, so war Nordeuropa in der plio-pleistozänen Übergangs- 
zeit (und wahrscheinlich auch im Oberpliozän) fraglos eines der dichtestbesiedelten Gebiete der Erde. Die umweltlich günstigsten Voraussetzungen für einen Aufenthalt des Menschen in Europa waren zu jener Zeit gegeben. Denn im gleichen Maße wie ein Absinken der Temperatur um einige Grade zu einer Vereisung im Norden führte, dürfte ein gleichhoher Temperaturanstieg über den heutigen Durchschnitt zu einer Verlagerung des subtropischen Gürtels nach Norden und vielleicht zu einer größeren Austrocknung weiter Gebiete des afrikanischen Hochlandes geführt haben. Reiche Funde an Fauna und Flora aus den entsprechenden Erdschichten bezeugen jedenfalls für Europa eine „üppige“ Umwelt.

$\mathrm{Da}$ der Mensch während der Auswirkung der frühpleistczänen glazialen Veränderungen Europa mindestens teilweise räumen mußte, scheint es nicht abwegig anzunehmen, daß die verdrängten "Ureuropäer“, vielleicht solche vom Heidelberger Typus, nicht unwesentlich zur Anreicherung der ältesten Geröllkulturen in Afrika beigetragen haben.

Nach den bisher erarbeiteten Erkenntnissen ist in Afrika bis heute kein Skelettrest eines Menschen gefunden worden, der älter wäre als der des Heidelbergers von Mauer, und auch die den Bifaceindustrien vorangehenden Kafuan-Kulturen in Afrika sind von nur frühpleistozänem Alter und nicht als dem Pliozän angehörig erwiesen.

Wenn wir nach dem heutigen Stande des Wissens feststellen können, daß Afrika nicht früher als Europa von Menschen bewohnt war, so liegt kein Anlaß zu der Annahme vor, daß Europa ursprünglich von Afrika aus besiedelt worden ist. Denn je weiter wir von der frühpleistozänen Grenze ins Pliozän hinabgehen, umso günstiger waren die allgemeinen Verhältnisse für einen Aufenthalt des Menschen in Europa. Wenn wir in Europa für die Zeit des plio-pleistozänen Überganges eine reichgegliederte, typologisch festgefügte Werkzeugindustrie eines Menschen festgestellt haben, so ist bei Beachtung der allgemeinen Gegebenheiten die Annahme berechtigt, daß der Träger dieser Industrie, als den wir den Heidelberger ansehen, auf Vorformen zurückgeht, die schon im Pliozän in Europa lebten.

Wenn wir versuchen, in Europa nach den Kulturresten eines pliozänen Urmenschen $\mathrm{zu}$ forschen, so sind wir heute nicht mehr darauf angewiesen, nach irgendwelchen eolithisch anmutenden Zufallsprodukten suchen $\mathrm{zu}$ müssen, die wir als typologisch isoliert stehende Abschläge, zweifelhafte Kratzer oder dergleichen genetisch nirgendwo anzuhängen vermögen. Wir stehen vielmehr an der pliozänen Grenze mit dem Wissen um eine Industrie, die ein festgefügtes Typengepräge trägt, im Sinne eines gewissen kulturellen Hochstandes. Um eine solche individuelle Industrie zu entwickeln, bedurfte es u. E. einer längeren Anlaufzeit, und wir sind der Auffassung, daß wir mit dem typologischen Leitfaden der Heidelberger Industrie beträglich weit ins Pliozän hinuntergelangen werden, vielleicht in Zeitabschnitte hinab, in denen die biologischen Voraussetzungen zur Entwicklung von Menschen in Eurasien gleich günstig oder günstiger waren als im Gürtel der heutigen Äquatorialgebiete.

$$
\mathrm{Nachtrag}
$$

Artefakte aus dem Oberpliozän von Sï 1 zfeld/Meiningen (Thür.)

Bei dem ersten Versuch, Artefakte aus oberpliozänen Ablagerungen zu erlangen, besuchte ich am 29. 6. 56 in Begleitung von Frl. Dr. Minna Lang eine Kiesgrube bei Sülzfeld nahe Meiningen in Thüringen. Der geringgroße, bis zu $6 \mathrm{~m}$ tiefe Aufschluß ist geologisch noch nicht näher untersucht und noch nicht publiziert worden. Als Hangendes liegen dort Flußschottern nur 0,5 m starke, fast steinfreie humose Böden auf. In den mit z. T. scharfkantigen Sandsteinschottern durchsetzten Flußgeröllen wurden u. a. Reste von Mastodon arvernensis, Mastodon borsoni und Waldnashorn aufgefunden (M. LANG 
1955). In einigen nicht sehr entfernt gelegenen gleichalten Aufschlüssen bei Jüchsen wurd.en u. a. neben dieser Fauna noch Tapir und oberpliozäne Hirsche aufgefunden, worauf wir in einer ausführlicheren Abhandlung eingehen werden. Wir schließen uns hier als Arbeitshypothese der von Experten vertretenen Auffassungen an, daß die Schotter von Sülzfeld dem Oberpliozän angehören (DiETrich 1953). Es würde aber auch eine frühestpleistozäne Ansetzung unsere Auffassung über die Bedeutung der vorgeschichtlichen Funde von Sülzfeld in keiner Weise ändern.

An der Oberfläche der in der Grube angereicherten ausgesiebten Schotter (die, als hangnahe anstehend, auch z. T. scharfkantige Sandsteinblöcke von $0,5 \mathrm{~m}$ Seitenlänge führen) fand ich bei diesem Besuch 40 Artefakte. Sie sind alle aus Sandstein angefertigt und zumeist vorzüglich, also scharfkantig erhalten. Typologisch geben die Geräte das Gesamtbild der Heidelberger Industrie von Mauer wieder. Neben Nasenschabern und Hobeln kommen u. a. auch Einzelformen (wie Rust 1956, Tafel 25) vor. Daneben führt Sülzfeld aber augenscheinlich auch Sonderformen, wie kurze sehr spitze Nasenschaber. Wir hoffen, nach einer ausgedehnteren Untersuchung des Sülzfelder Vorkommens in Kürze anhand einiger hundert Artefakte ein besser orientiertes Bild vom Typenbestand der Sülzfelder Industrie geben zu können.

Die Bedeutung der Sülzfelder Artefakte ist in der Feststellung zu sehen, daß wir damit zur Kenntnis einer Industrie gelangt sind, die sich am Ende des Tertiärs als rypologisch fest begründet und in den Einzelformen als einem geregelten, handwerk* lich straff geordneten Spezialwerkzeugbestand eingefügt ausweist! Die Heidelberger Industrie vom Typus Sülzfeld ist weltweit von jenem Stand der kulturellen Außerungen der frühesten Menschheit entfernt, die wir als dem Eolithikum zugehörig vielleicht einmal auffinden werden.

$\mathrm{Zu}$ unserer mehrfach vorgebrachten hypothetischen Auffassung, daß die Anlaufzeit zur Entwicklung einer typologisch hochgeschraubten Industrie, wie unserer Heidelberger Kultur, beträchtlich lang gewesen sein muß, können wir als praktisches Beispiel jetzt auch die Zeitspanne zwischen Mauer und Sülzfeld anführen. Diese nicht oder nur in wenigen Sonderformen typologisch voneinander abtrennbaren Industrien weisen einen Altersunterschied von 100000 oder mehreren hunderttausend Jahren auf. Da in der Frühzeit weder an körperbauliche noch an geistigkulturelle mutative Großsprünge zu denken ist, sind wir daher der Auffassung, daß es gelingen wird, in Europa Kulturreste von Prä-Heidelberg-Industrien (und damit auch Körperreste ihres Trägers) aufzufinden, die 2 oder mehrere Millionen Jahre zurückgehen. Wir sehen bisher keine Hinweise, die erwägen ließen, daß der Artefakte führende Mensch im Pliozän oder Miozän aus Afrika oder Asien nach Europa eingewandert sein müßte.

Das oberpliozäne Vorkommen von Sülzfeld festigt ferner die Basis zur gedanklichen Auffassung, daß auch Nordeuropa im Endtertiär bewohnt war. Wir sind der Ansicht, daß es gelingen wird, dieser Auffassung den hypothetischen Charakter zu nehmen. Diesbezügliche Möglichkeiten wird eine ausgeweitete Einsichtnahme in den Typenbestand von Sülzfeld als Grundlage für Vergleichsobjekte zum Norden geben. Ferner will uns scheinen, daß nicht alle Heidelberger Industrien im Norden von gleichem Alter sind. In einzelnen geschlossenen Vorkommen dieser Art kommen häufig Artefakte vor, die aus eisgeschrammten Geschieben hergestellt sind. Sie sind vermutlich in einem frühpleistozänen Interglazial angefertigt. In anderen Artefaktkomplexen finden sich keine geschrammten Geräte. Solche dürften (in Verbindung mit kennzeichnenden typologischen Eigenarten) als dem Oberpliozän angehörig aufzufassen sein. Möglicherweise bieten auch die unterschiedlich stark ausgeprägten Windschliffe Hilfsstellung bei den Datierungen.

Durch eine zweite, vom 27.-28. 6. 56 in Gemeinschaftsarbeit mit Prof. BEHM* BLANCKE und seinen Mitarbeitern vom Institut für Urgeschichte Weimar in Sülzfeld 
durchgeführte Untersuchung konnte nachgewiesen werden, daß die Artefakte u. a. an Schichten gebunden sind, die 4-5 $\mathrm{m}$ unter der heutigen Oberfläche anstehen. Es liegen jetzt über 100 vorzügliche Geräte vor. Die oberpliozänen Schotter von Sülzfeld sind ungemein reich an Artefakten, und ich schätze das dortige Vorkommen (wie dasjenige in der Neckarschleife bei Mauer) auf einige hunderttausend Stücke. Eine informative Exkursion ließ deutlich werden, daß die dem Sülzfelder Raum benachbart gelegene Rhön einen außerordentlichen Reichtum an Basaltartefakten vom Heidelberger Typus auf weist.

Zusammenfassend glauben wir den bisherigen Forschungsergebnissen folgende Erkenntnisse abgewinnen zu können. Mittel- und Nordeuropa waren im Mittelpleistozän verhältnismäßig schwach besiedelt. Im Frühpleistozän war die Besiedlung dichter. Am stärksten war die Population im Oberpliozän. Die zu Millionen auch im Bereich des fennoskandischen Vereisungsgebietes eingeschlossenen tertiärzeitlichen Artefakte werden erweisen, daß Nordeuropa (und z. T. wohl auch Nordasien) zu dieser Zeit eines der dichtestbesiedelten Gebiete der Erde war.

\section{Literaturverzeichnis}

Dietrich, W. O.: Neue Funde des etruskischen Nashorns in Deutschland und die Frage der Villafranchium-Faunen. - Geologie 2, S. 417-430. Berlin 1953.

GripP, K.: Ưber die äußerste Grenze der letzten Vereisung in Nordwestdeutschland. - Mitt. geograph. Ges. Hamburg 35, 1924.

LANG, M.: Auf Spuren oberpliozäner Mastodonten. - Unsere Heimat. Neue Beiträge zur Naturund Heimatforschung im Bezirk Suhl, 1. Jahrg., Heft 1, Suhl 1955.

Lütтig, G.: Alt- und mittelpleistozäne Eisrandlagen zwischen Harz und Weser. - Geolog. Jb. 70, Hannover 1954.

Rern, U.: Die pollenstratigraphische Gliederung des Pleistozäns in Norddeutschland. - Eiszeitalter und Gegenwart 6, Óhringen 1955.

Rust, A.: Artefakte aus der Zeit des Homo heidelbergensis in Süd- und Norddeutschland. Habelt, Bonn 1956.

Soergel, W.: Die diluvialen Terrassen der Ilm. - Jena 1924.

Wolters, R.: Ausbildung und Lagerung der plio-pleistozänen Grenzschichten im niederrheinischen Grenzgebiet von Niederkrüchten-Brüggen. - Geolog. Jb. 69, Hannover 1954.

Woldstedt, P.: Norddeutschland und angrenzende Gebiete im Eiszeitalter. - 2. Aufl., Stuttgart 1955.

Manuskr. eingeg. 5. 6. 1956.

Anschrift d. Verf.: Dr. Alfred Rust, Ahrensburg/Holstein, Am Rehm 52. 